\title{
Nocardia farcinica Brain Abscess in an Immunocompetent Host With Pulmonary Alveolar Proteinosis: A Case Report and Review of the Literature
}

\author{
Sarah E. Grond ${ }^{1}$, Alyssa Schaller ${ }^{2}$, Alexandra Kalinowski ${ }^{1}$, Kimberly A. Tyler ${ }^{1}$, Pinky Jha ${ }^{1}$ \\ 1. Internal Medicine, Medical College of Wisconsin, Wauwatosa, USA 2. Pharmacy, Concordia University of Wisconsin, \\ Mequon, USA
}

Corresponding author: Sarah E. Grond, sgrond@mcw.edu

\begin{abstract}
A 55-year-old immunocompetent male presented with new-onset seizures and acute respiratory failure requiring intubation and a stay in the medical intensive care unit. Magnetic Resonance Imaging (MRI) of the brain revealed ring-enhancing lesions, and Computed Tomography (CT) chest showed ground-glass opacity. The patient underwent craniotomy and bronchoscopy, followed by culture of the purulent aspirate from lesions in the brain and bronchoalveolar lavage (BAL). After extensive infectious workup, the patient was diagnosed with a Nocardia farcinica brain abscess plus underlying pulmonary alveolar proteinosis (PAP). Based on a recommendation from an infectious disease expert, the patient was treated with trimethoprimsulfamethoxazole (TMP/SMX).
\end{abstract}

This case highlights the importance of understanding that, though rare, infections such as nocardiosis can present in immunocompetent patients and cause severe morbidity.

Categories: Internal Medicine, Infectious Disease

Keywords: pulmonary alveolar proteinosis, nocardia species, disseminated nocardiosis, brain abscess

\section{Introduction}

Nocardia species are a group of obligate aerobic soil saprophytes that belong to the order Actinomycetales. They are weakly acid-fast bacilli that form branching filaments, structures that resemble the hyphae formed by fungi, and can often be deceiving on culture [1,2]. Over 80 Nocardia species have been identified, and roughly half of those organisms are clinically significant. Nocardia asteroides has historically been implicated most often in human disease; however, it is now divided into six separate species and renamed $N$. asteroides complex, of which $N$. abscessus, $N$. nova, and $N$. farcinica are some of the most clinically relevant human pathogens [3]. N. farcinica has been shown to have greater virulence than other Nocardia species, and it is commonly implicated in systemic infection [3]. N. brasilensis is another common human pathogen that most often causes cutaneous infections [3].

Review began 10/04/2020 Review ended 11/12/2020 Published 11/15/2020

\section{() Copyright 2020}

Grond et al. This is an open access article distributed under the terms of the Creative Commons Attribution License CC-BY 4.0., which permits unrestricted use, distribution, and reproduction in any medium, provided the original author and source are credited.
Every year about 500 to 1000 new cases of Nocardia infections are reported, most often in patients who have preexisting immune system compromise [4,5]. The average age of infection onset is 40 , and infections typically occur more often in males than females. Nocardia species are opportunistic pathogens; therefore, immunocompromised patients with conditions such as diabetes, cancer, lupus, and inflammatory bowel disease (IBD), are more at risk for infection [5]. Nocardia species can be found in water, soil, and decaying plants, as well as in dust and swimming pools, and transmission occurs via inhalation of the microorganisms or through skin inoculation through open wounds [1,2].

Here we report a case of a Nocardia farcinica brain abscess in an immunocompetent host with pulmonary alveolar proteinosis (PAP).

\section{Case Presentation}

A 51-year-old African American male with a medical history of psychosis, hypertension, and alcohol abuse presented with new-onset seizures, manifesting as repetitive movements of the left upper extremity. On review of systems, the patient also endorsed recent subjective fevers, cough, shortness of breath, and a 40to 50-pound weight loss over the last four months. Neurology was consulted for the status epilepticus, and workup included electroencephalogram (EEG), computed tomography (CT), and magnetic resonance imaging (MRI). Brain imaging revealed a $3.1 \times 2.3 \mathrm{~cm}$ mass in the right parietal lobe with surrounding edema, described as a ring-enhancing lesion (Figure 1). Significant laboratory findings included respiratory acidosis, lactic acid of $15.4 \mathrm{mmol} / \mathrm{L}$, elevated ferritin of $833 \mathrm{ng} / \mathrm{mL}$, and elevated lactate dehydrogenase (LDH) of 390 unit/L (Table 1). Subsequent chest x-ray (CXR) revealed a diffuse interstitial prominence. Chest imaging, along with respiratory symptoms, raised suspicion for COVID-19; however, viral swabs were 


\section{Cureus}

negative.

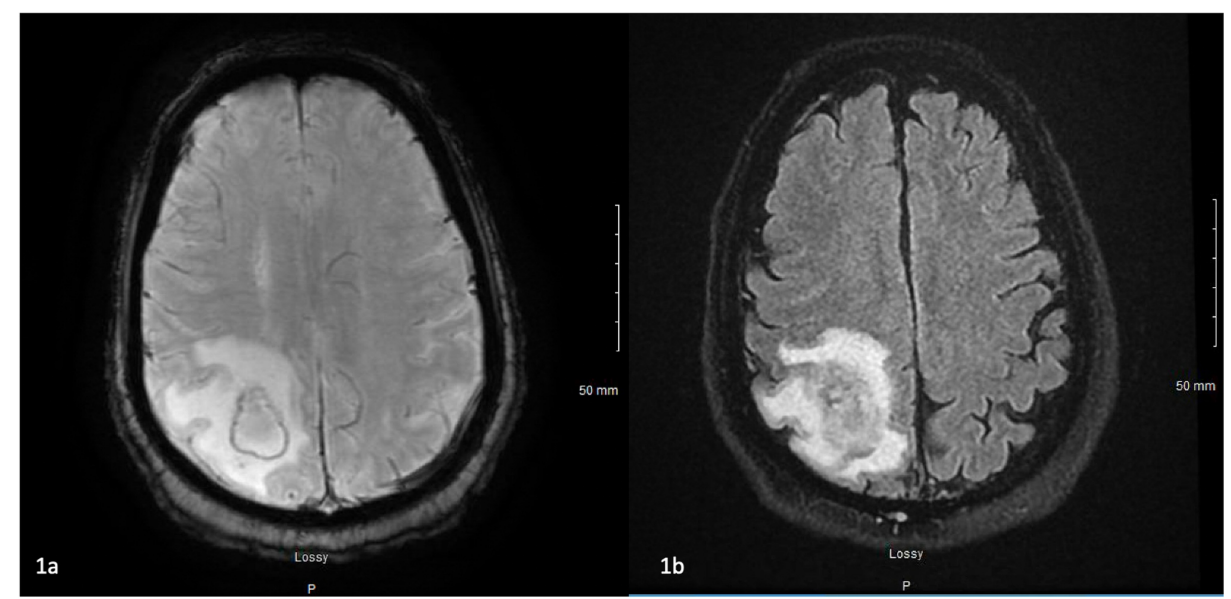

FIGURE 1: Initial MRI head showing $3.1 \times 2.3 \mathrm{~cm}$ ring-enhancing lesion in the right parietal lobe

Figure 1a: axial susceptibility-weighted imaging (SWI), Figure 1b: axial fluid-attenuated inversion-recovery (FLAIR) fat-suppressed (FS) imaging

\begin{tabular}{|c|c|}
\hline Test Name & Test Results (Reference Range) \\
\hline $\begin{array}{l}\text { White Blood } \\
\text { Cell Count }\end{array}$ & 9.8 (3.9-11.2 x 10e3/uL) \\
\hline $\begin{array}{l}\text { Red Blood Cell } \\
\text { Count }\end{array}$ & 4.9 (4.4-5.9 x 10e6/uL) \\
\hline Hemoglobin & 16.1 (13.7-17.5 g/dL) \\
\hline Hematocrit & $50(40-51 \%)$ \\
\hline $\begin{array}{l}\text { Mean } \\
\text { Corpuscular } \\
\text { Volume }\end{array}$ & 103 (79-98 fL) \\
\hline $\begin{array}{l}\text { Mean } \\
\text { Corpuscular } \\
\text { Hemoglobin }\end{array}$ & $33.1(25.7-32.2 \mathrm{pg})$ \\
\hline $\begin{array}{l}\text { Mean } \\
\text { Corpuscular } \\
\text { Hemoglobin } \\
\text { Concentration }\end{array}$ & 32 (32-36 g/dL) \\
\hline $\begin{array}{l}\text { Red Cell } \\
\text { Distribution } \\
\text { Width }\end{array}$ & $12.5(11-14.9 \%)$ \\
\hline Platelet Count & 287 (165-366 x 10e3/uL) \\
\hline $\begin{array}{l}\text { Mean Platelet } \\
\text { Count }\end{array}$ & 9.4 (9.0-11.8 fL) \\
\hline $\begin{array}{l}\text { Automated } \\
\text { Nucleated Red } \\
\text { Blood Cells }\end{array}$ & $0(0-0 \%)$ \\
\hline $\begin{array}{l}\text { Blood Urea } \\
\text { Nitrogen }\end{array}$ & $6(6-23 \mathrm{mg} / \mathrm{dL})$ \\
\hline Sodium & 143 (136-145 mmol/L) \\
\hline
\end{tabular}




\section{Cureus}

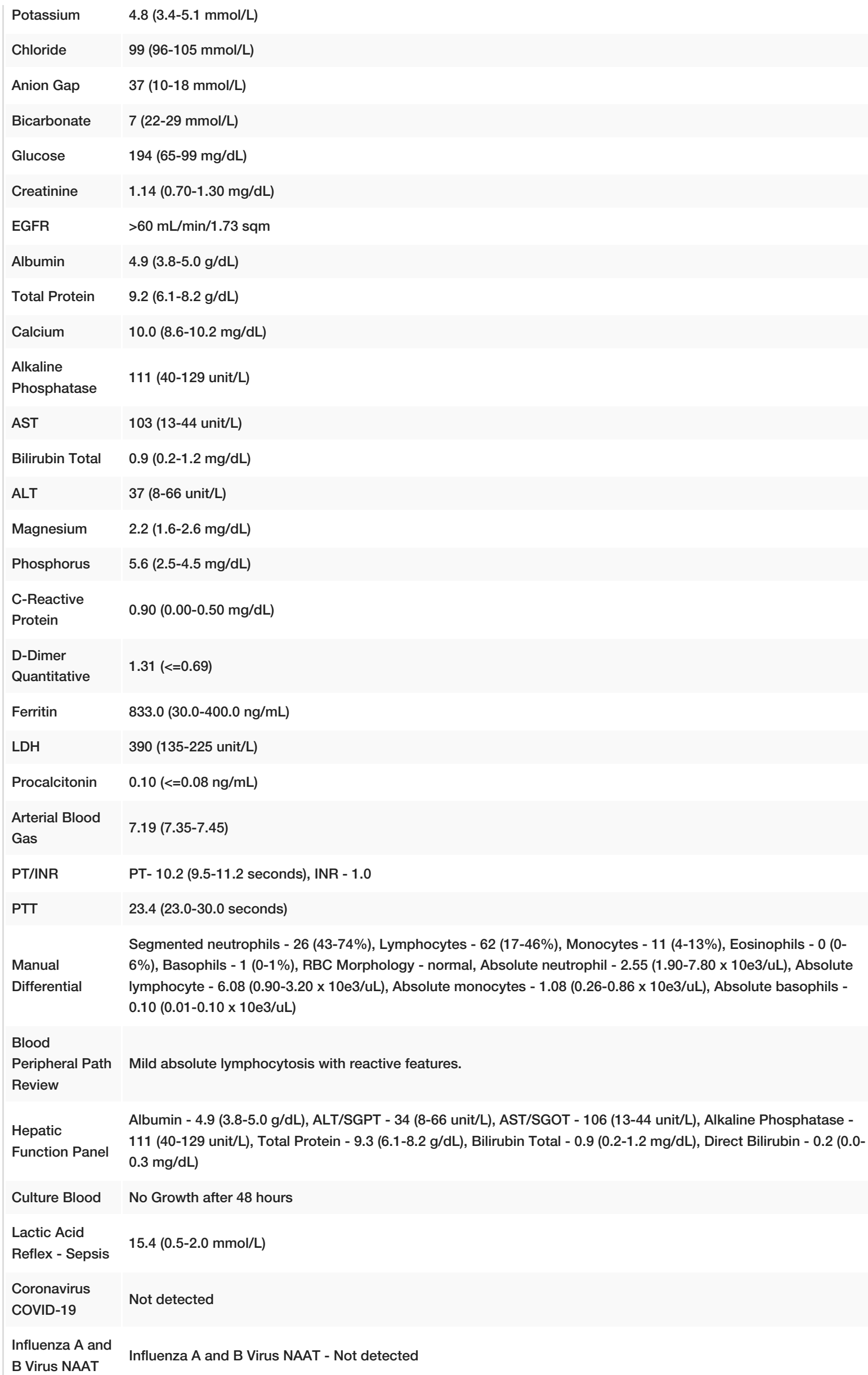




\section{Cureus}

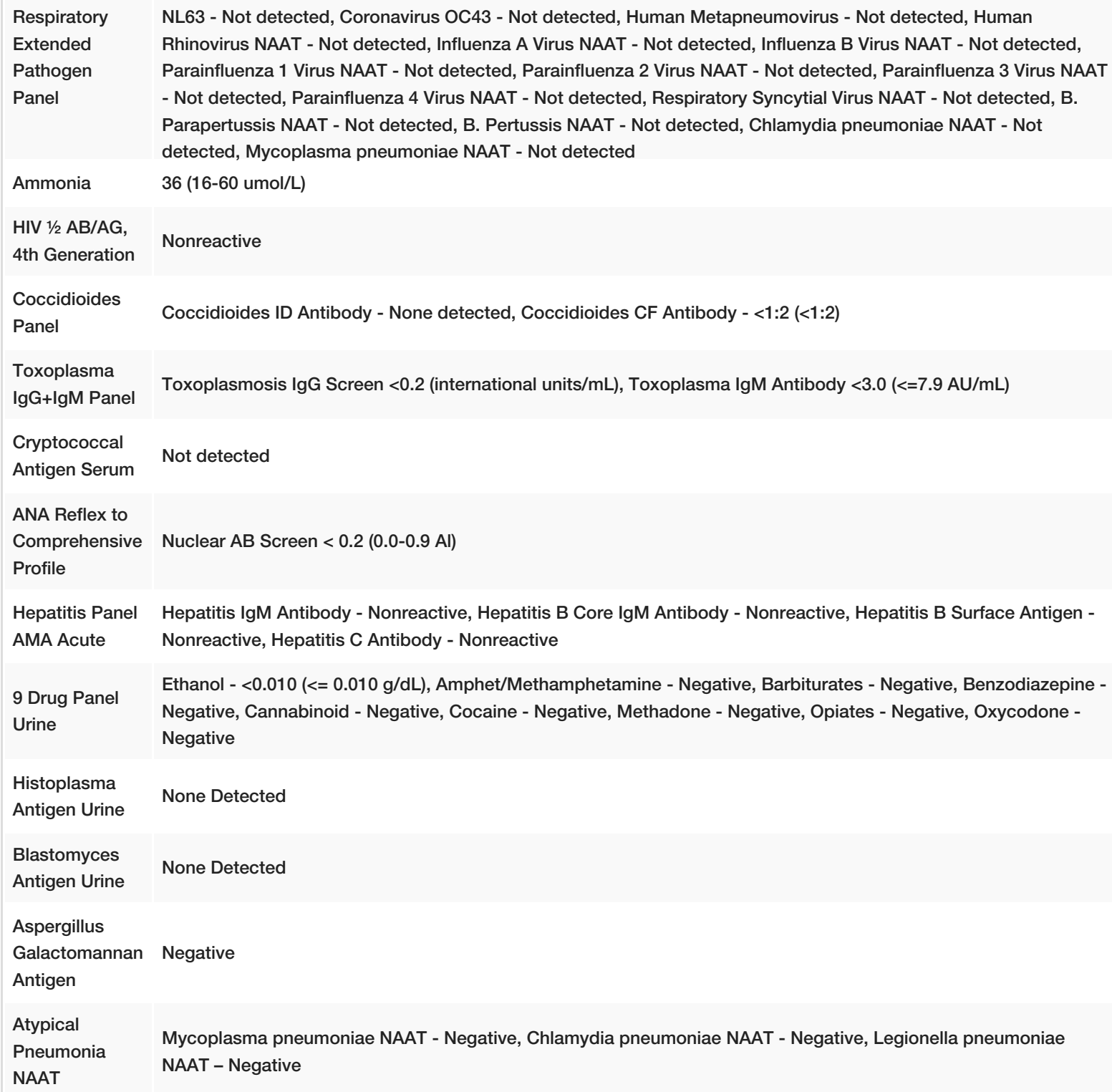

\section{TABLE 1: Initial laboratory workup, including reference ranges}

EGFR = estimated glomerular filtration rate, $\mathrm{AST}=$ aspartate aminotransferase, $\mathrm{ALT}=$ alanine aminotransferase, $\mathrm{LDH}=\mathrm{lactate}$ dehydrogenase, $\mathrm{PT}=$ prothrombin time, INR = International Normalized Ratio, PTT = partial thromboplastin time, SGPT = serum glutamic-pyruvic transaminase, SGOT = serum glutamic-oxaloacetic transaminase, NAAT = nucleic acid amplification test, $A U=$ arbitrary unit, $A B=$ antibody, $A G=$ antigen, $A N A=$ antinuclear antibody, AMA = American Medical Association, ID = immunodiffusion, $C F=$ complement fixation

Seizure control was initially attempted with levetiracetam, divalproex sodium, and fosphenytoin but required escalation to lacosamide and phenobarbital. On day one of hospitalization, the patient had worsening lethargy and mental status, so the patient was intubated. A decision was made to proceed with needle biopsy of the brain abscess, which revealed purulent fluid that grew branching, gram-positive rods, which were later confirmed to be Nocardia farcinica. An infectious disease expert was consulted, and the patient was started on cefepime, trimethoprim-sulfamethoxazole (TMP-SMX), and metronidazole for coverage of Nocardia, Blastomycosis, and Cryptococcus species.

Bronchoscopy with bronchoalveolar lavage (BAL) was performed due to the observation of ground-glass opacities on CT (Figure 2). Secretions were thick and purulent with a "milky" appearance, and a gram stain resulted in rare gram-negative rods and gram-positive cocci. Periodic acid-Schiff (PAS) stain was positive. The findings were consistent with PAP. Given the diagnosis of Nocardia farcinica brain abscess, additional infectious workup was ordered, and no other infectious pathogens were identified, including negative human immunodeficiency virus (HIV) test (Table 1). Due to the suspected PAP in the setting of acute hypoxemic respiratory failure, a right whole lung lavage was performed. Post-operatively the patient 


\section{Cureus}

developed intermittent fevers, and linezolid and moxifloxacin were added for additional coverage. Blood cultures were sent and had no growth. Given the patient's clinical improvement, antibiotics were narrowed back to TMP-SMX.

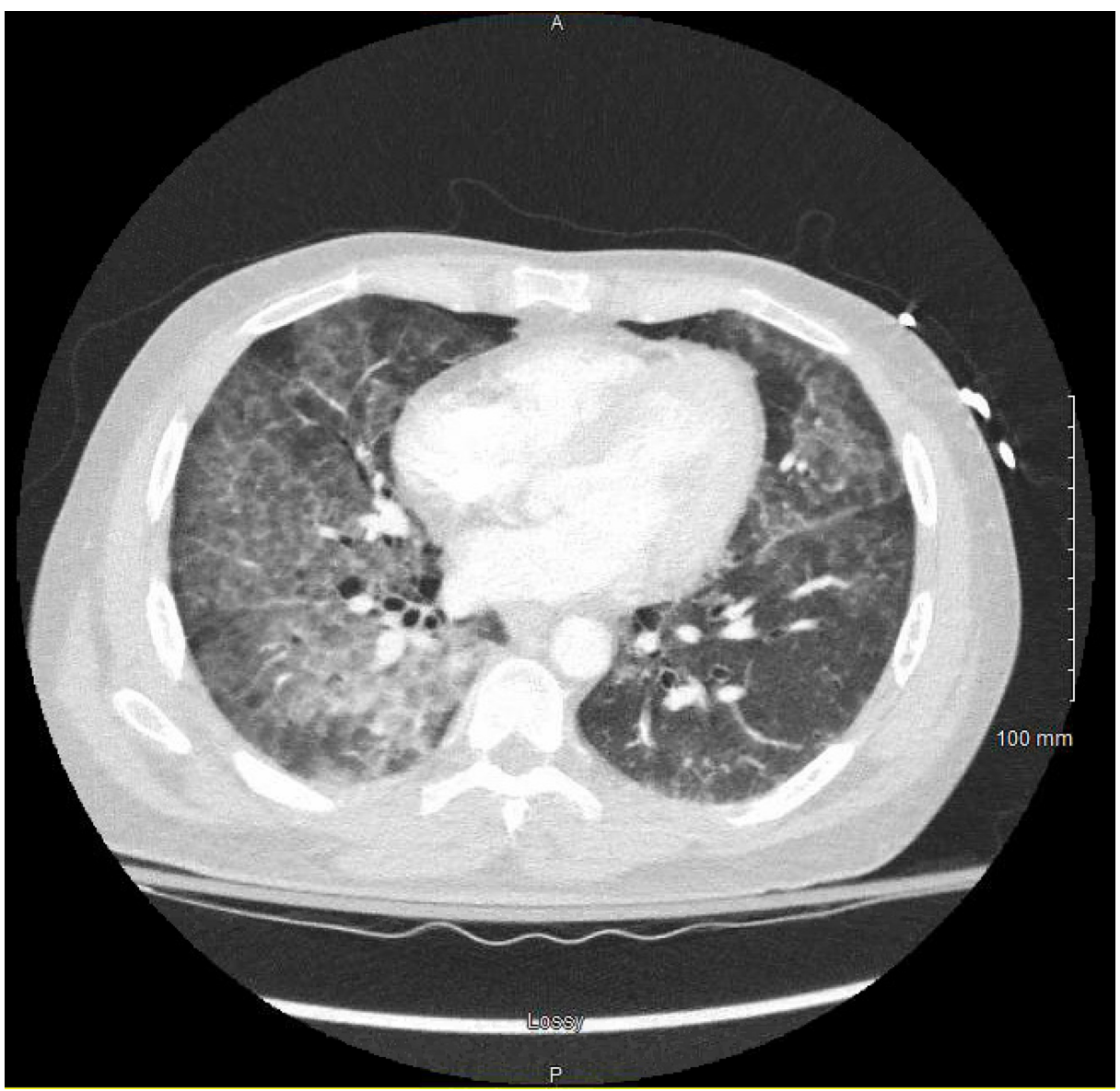

FIGURE 2: Chest CT showing ground-glass opacities

Surveillance MRI on hospital day 13 showed an increase in the previously drained right parietal abscess. Antibiotics were escalated to oral TMP-SMX, intravenous (IV) metronidazole, ceftriaxone, and vancomycin. A repeat craniotomy was performed for drainage, and Nocardia farcinica continued to be the only pathogen present. Antibiotics were narrowed back down to TMP-SMX. At this point, the patient experienced another seizure, requiring adjustments of his levetiracetam and phenobarbital. The patient became febrile once again, and antibiotics were switched to IV TMP-SMX, linezolid $600 \mathrm{mg}$ every 12 hours, and moxifloxacin 400 $\mathrm{mg}$ four times a day. Soon after, the patient was noted to have increased secretions and became increasingly lethargic and unarousable, requiring intubation again. The left whole lung lavage was scheduled for suspected PAP and proceeded as planned. The patient was successfully extubated soon after, and antibiotics were tapered back down to TMP-SMX.

By week three of hospitalization, a repeat MRI showed improvement of the cerebral abscess and edema. The patient continued to improve clinically and was discharged to neurologic rehabilitation on hospital day 34 with plans for weekly basic metabolic panels (BMPs) and two double-strength TMP-SMX three times daily for one year, in addition to continuing dexamethasone $4 \mathrm{mg}$ twice a day, clobazam $10 \mathrm{mg}$ twice a day, lacosamide $200 \mathrm{mg}$ twice a day, levetiracetam $1250 \mathrm{mg}$ twice a day, $\mathrm{NaCl} 2 \mathrm{~g}$ three times daily.

Six weeks post-discharge, follow-up CT showed worsening bilateral ground-glass opacities with interlobular septal thickening and new consolidated, bilateral opacities. The patient was admitted for scheduled bronchoalveolar lavages, which proceeded as planned without complication.

\section{Discussion}

Nocardia species are a group of obligate aerobic soil saprophytes that most often cause pulmonary, central nervous system, and cutaneous infection in immunocompromised hosts [1]. Nocardiosis, though rare, can cause severe disease and widespread disseminated infection. Though Nocardia species are commonly found in soil, infection rates are low. Additionally, the majority of infections are caused by inhalation, and 
therefore pulmonary involvement is the most likely initial presentation [4]. Other typical manifestations include brain abscesses and skin/soft tissue infection. The majority of Nocardia infections are diagnosed in immunocompromised hosts; however, at least $35 \%$ of patients are immunocompetent at the time of diagnosis [1].

Immunocompetence is defined as having an appropriate immune response following exposure to a foreign antigen. Since pulmonary alveolar proteinosis (PAP) is not considered an immunodeficiency, the patient was determined to be immunocompetent in absence of immunodeficiency. Here we report a case of a Nocardia farcinica brain abscess and PAP in an otherwise immunocompetent host.

We conducted an extensive literature search of Nocardia farcinica infections in immunocompetent hosts. Eighteen previous cases of $N$. farcinica in immunocompetent hosts were found, and those between 2010 and 2020 can be seen in Table 2. We did not find any case reports that highlighted Nocardia farcinica infections in patients with PAP.

\begin{tabular}{|c|c|c|c|}
\hline Authors, Year of Study & Nocardia dissemination & Immunocompetent? & Other comorbidities \\
\hline Vuotto et al., 2011 [6] & Iliac artery stent infection & Yes & lliac artery stenosis \\
\hline Budzik et al., 2012 [7] & Pneumonia and synovitis & Yes & One time steroid injection in knee \\
\hline Kim et al., 2014 [8] & Brain abscess & Yes & HTN \\
\hline Kim et al., 2016 [9] & Pneumonia, mediastinitis & Yes & HTN \\
\hline Boamah et al., 2016 [10] & Pneumonia, brain abscess & Yes & Smoking, GERD \\
\hline Pascual-Gallego et al., 2016 [11] & Brain abscess (cerebellum) & Yes & $\begin{array}{l}\text { Olfactory groove meningioma } 11 \\
\text { years earlier }\end{array}$ \\
\hline Jackson et al., 2017 [12] & $\begin{array}{l}\text { Pneumonia, adrenal abscess, brain } \\
\text { abscess }\end{array}$ & Yes & CAD, smoking \\
\hline Chaudhari et al., 2017 [13] & Brain abscess & Yes & Diabetes (well-controlled) \\
\hline Holmes et al., 2018 [14] & Brain abscess & Yes & COPD, HTN \\
\hline Faircloth and Troy, 2019 [15] & Pericardial effusion and pericarditis & Yes & Alcohol consumption \\
\hline Wang et al., 2019 [16] & Orbital infection & Yes & None \\
\hline $\begin{array}{l}\text { Moniuszko-Malinowska et al., } \\
2020 \text { [17] }\end{array}$ & Chronic meningitis & Yes & None \\
\hline
\end{tabular}

TABLE 2: Literature review of Nocardia farcinica infections in immunocompetent hosts between 2010-2020

$\mathrm{HTN}=$ hypertension, GERD= gastroesophageal reflux disease, $\mathrm{CAD}=$ coronary artery disease, COPD=Chronic Obstructive Pulmonary Disease

The clinical presentation of Nocardia infections varies depending on organ system involvement; however, the symptoms tend to be nonspecific and require further workup. The most common infections are cutaneous, pulmonary, and neurological; however, kidney, spleen, liver, bone, and joint infections may also occur [1]. Patients may present with fever, weight loss, night sweats, cough, or chest pain, but they can also present with headaches and seizures if there is neurological involvement [4]. Patients with cutaneous infections will often present with erythema, nodules, or ulcers [1]. Prior to diagnosis, a full patient history should be obtained along with proper imaging. This imaging should include CT, chest X-ray, and MRI, along with appropriate neurological imaging and assessment to rule out any central nervous system (CNS) involvement. Specimen collection can include biopsy, bronchoscopy, aspiration, as well as sputum collection, and a Gram stain and modified acid-fast stain should be used for culturing [5].

About $39 \%$ of patients present with pulmonary Nocardia infection; however, approximately $32 \%$ of those cases disseminate to other organ systems [1]. Patients with pulmonary involvement often present with necrotizing pneumonia, which can lead to pleural effusions, empyemas, as well as pericarditis. Over $44 \%$ of all cases have CNS involvement, which is most often secondary to pulmonary infection [1]. Patients who are immunocompromised may have asymptomatic abscesses that exhibit a latency period for up to three years; however, when these patients present with symptoms, the infection is typically disseminated and more lifethreatening. Additionally, primary cutaneous and soft tissue nocardiosis can occur as a result of direct 
inoculation of the skin [18]. There are three types of cutaneous infections, including cellulitis, actinomycetomas, and lymphocutaneous disease. Actinomycetomas involve bone and subcutaneous tissue and typically have a late-onset after infection, whereas lymphocutaneous disease results in pus, drainage, and crusting of lesions and can lead to lymphatic abscesses $[1,18]$.

There are currently no formal treatment guidelines for managing Nocardia infections, which highlights the need for future research and the development of treatment protocols based on randomized controlled trials. Treatment of Nocardia infections is highly individualized, and susceptibility testing is key as antimicrobial resistance is common among different Nocardia species [19]. Monotherapy is typically sufficient for immunocompetent hosts with systemic or cutaneous infection; however, in immunocompromised patients or in patients with pulmonary or disseminated infection, double or triple therapy is often warranted [5]. The duration of therapy varies widely, and individual patient factors must be considered. Immunocompetent patients with cutaneous infection may need only a course of one to three months of antibiotic therapy, whereas uncomplicated pulmonary infections may require treatment for less than six months. In immunocompromised patients, patients with disseminated infection, or patients with CNS involvement, a minimum of six months of antimicrobial therapy is typically used, and patients are monitored thereafter for infection recurrence [5]. In addition, surgical management may be necessary, especially in those with severe disease and failure of antimicrobial therapy $[1,20]$.

Various drug susceptibility analyses have been performed. Sulfonamides, aminoglycosides, betalactam/beta-lactamase inhibitors, fluoroquinolones, macrolides, and tetracyclines have all been explored as treatment options for Nocardia infections. Sulfonamide antimicrobials, such as TMP-SMX, are the treatment agents of choice. Nocardia farcinica has been noted to have increased antibiotic resistance compared to other strains, with resistance to beta-lactams and aminoglycosides but susceptibility to amoxicillinclavulanic acid, amikacin, moxifloxacin, linezolid, and TMP-SMX and variable susceptibility toward imipenem and ciprofloxacin [19]. Before antibiotic susceptibility tests return, empiric therapy of TMP-SMX, amikacin, and either ceftriaxone or imipenem is usually initiated [3].

To the best of our knowledge, this case report is the first case of a Nocardia farcinica brain abscess in a patient with underlying PAP.

\section{Conclusions}

In conclusion, we present a case of a Nocardia farcinica brain abscess in an immunocompetent patient with pulmonary alveolar proteinosis. With this case, we hope to increase awareness among practitioners about this challenging diagnosis, especially in immunocompetent hosts. Additionally, Nocardia farcinica is a rare and potentially fatal infection that is difficult to treat and often requires a multidisciplinary approach.

\section{Additional Information \\ Disclosures}

Human subjects: Consent was obtained by all participants in this study. Conflicts of interest: In compliance with the ICMJE uniform disclosure form, all authors declare the following: Payment/services info: All authors have declared that no financial support was received from any organization for the submitted work. Financial relationships: All authors have declared that they have no financial relationships at present or within the previous three years with any organizations that might have an interest in the submitted work. Other relationships: All authors have declared that there are no other relationships or activities that could appear to have influenced the submitted work.

\section{References}

1. Henderson NM, Sutherland RK: Nocardia and Actinomyces. Medicine (Baltimore). 2017, 45:753-756. 10.1016/j.mpmed.2017.09.012

2. Beaman BL, Beaman L: Nocardia species: host-parasite relationships. Clin Microbiol Rev. 1994, 7:213-264. 10.1128/CMR.7.2.213

3. Brown-Elliott BA, Brown JM, Conville PS, Wallace RJ: Clinical and laboratory features of the Nocardia spp. based on current molecular taxonomy. Clin Microbiol Rev. 2006, 19:259-282. 10.1128/CMR.19.2.259282.2006

4. Nocardiosis | CDC. (2018). Accessed: September 1, 2020: https://www.cdc.gov/nocardiosis/index.html.

5. Rawat D, Rajasurya V, Chakraborty RK, Sharma S: Nocardiosis. In: StatPearls [Internet]. StatPearls Publishing, Treasure Island (FL); 2020.

6. Vuotto F, Faure K, Queyre V, et al.: Vascular nosocomial Nocardia farcinica infection after arterial stenting in an immunocompetent patient. Can J Infect Dis Med Microbiol. 2011, 22:10-11. 10.1155/2011/216873

7. Budzik JM, Hosseini M, Mackinnon AC, Taxy JB: Disseminated Nocardia farcinica: literature review and fatal outcome in an immunocompetent patient. Surg Infect. 2012, 13:163-170. 10.1089/sur.2011.012

8. Kim S, Lee KL, Lee DM, et al.: Nocardia brain abscess in an immunocompetent patient . Infect Chemother. 2014, 46:45-49. 10.3947/ic.2014.46.1.45

9. Kim J, Kang M, Kim J, et al.: A case of Nocardia farcinica pneumonia and mediastinitis in an immunocompetent patient. Tuberc Respir Dis. 2016, 79:101-103. 10.4046/trd.2016.79.2.101

10. Boamah H, Puranam P, Sandre RM: Disseminated Nocardia farcinica in an immunocompetent patient 


\section{Cureus}

IDCases. 2016, 6:9-12. 10.1016/j.idcr.2016.08.003

11. Pascual-Gallego M, Alonso-Lera P, Arribi A, Barcia JA, Marco J: Nocardia farcinica abscess of the cerebellum in an immunocompetent patient: a case report and review of the literature. Asian J Neurosurg. 2016, 11:454-455. 10.4103/1793-5482.145179

12. Jackson C, McCullar B, Joglekar K, Seth A, Pokharna H: Disseminated Nocardia farcinica pneumonia with left adrenal gland abscess. Cureus. 2017, 9:e1160. 10.7759/cureus.1160

13. Chaudhari DM, Renjen PN, Sardana R, Butta H: Nocardia farcinica brain abscess in an immunocompetent old patient: a case report and review of literature. Ann Indian Acad Neurol. 2017, 20:399-402. 10.4103/aian.AIAN_263_17

14. Holmes DN, Armstrong L, Bennett J, Simms HN: Neurosurgical management of multiple intracranial Nocardia and Enterococcus abscesses in an immunocompetent patient. J Surg Case Rep. 2018 , 2018:rjy015. 10.1093/jscr/rjy015

15. Faircloth EL, Troy P: A rare presentation of Nocardia pericarditis leading to cardiac tamponade in an immunocompetent patient. Cureus. 2019, 11:e4140. 10.7759/cureus.4140

16. Wang A, Xu Q, Wang Y, Liao H: Orbital and intracranial Nocardia farcinica infection caused by trauma to the orbit: a case report. BMC Infect Dis. 2019, 19:953. 10.1186/s12879-019-4605-Z

17. Moniuszko-Malinowska A, Czupryna P, Swiecicka I, et al.: Nocardia farcinica as a cause of chronic meningitis - case report. BMC Infect Dis. 2020, 20:56. 10.1186/s12879-020-4764-y

18. Wilson JW: Nocardiosis: updates and clinical overview . Mayo Clin Proc. 2012, 87:403-407. 10.1016/j.mayocp.2011.11.016

19. Hamdi AM, Fida M, Deml SM, Abu Saleh OM, Wengenack NL: Retrospective Analysis of Antimicrobial Susceptibility Profiles of Nocardia Species from a Tertiary Hospital and Reference Laboratory, 2011 to 2017. Antimicrob Agents Chemother. 2020, 64:e01868-19. 10.1128/AAC.01868-19

20. LoCicero J, Shaw JP, Lazzaro RS: Surgery for other pulmonary fungal infections, Actinomyces, and Nocardia . Thorac Surg Clin. 2012, 22:363-374. 10.1016/j.thorsurg.2012.04.002 